\title{
Oral Glucose Tolerance Testing identifies HIV+ infected women with Diabetes Mellitus (DM) not captured by standard DM definition
}

\author{
Sophie Seang ${ }^{1 * \#}$, Jordan E Lake ${ }^{1 \#}$, Fang Tian², Kathryn Anastos ${ }^{3}$, Mardge H Cohen ${ }^{4}$ and Phyllis C Tien ${ }^{1}$ \\ ${ }^{1}$ Department of Medicine, Division of Infectious Diseases, University of California, Los Angeles, California, USA \\ ${ }^{2}$ Anthem, Inc., Alexandra, VA, USA \\ ${ }^{3}$ Department of Medicine and Epidemiology and Population Health, Albert Einstein College of Medicine and Montefiore Medical Center, Bronx, New York, USA \\ ${ }^{4}$ Departments of Medicine, Cook County Health \& Hospital System, and Rush University, Chicago, Illinois, USA \\ "Both the authors equally contributed to the work
}

\begin{abstract}
Objective: HIV-infected (HIV+) individuals may have differential risk of diabetes mellitus (DM) compared to the general population, and the optimal diagnostic algorithm for DM in HIV+ persons remains unclear. We aimed to assess the utility of oral glucose tolerance testing (OGTT) for DM diagnosis in a cohort of women with or at risk for HIV infection.

Methods: Using American Diabetic Association DM definitions, DM prevalence and incidence were assessed among women enrolled in the Women's Interagency HIV Study. DM was defined by 2-hour OGTT $\geq 200 \mathrm{mg} / \mathrm{dL}$ (DM_ OGTT) or a clinical definition (DM_C) that included any of the following: (i) anti-diabetic medication use or self-reported DM confirmed by either fasting glucose (FG) $\geq 126 \mathrm{mg} / \mathrm{dL}$ or $\mathrm{HbA} 1 \mathrm{c} \geq 6.5 \%$, (ii) $\mathrm{FG} \geq 126 \mathrm{mg} / \mathrm{dL}$ confirmed by a second FG $\geq 126 \mathrm{mg} / \mathrm{dL}$ or $\mathrm{HbA} 1 \mathrm{c} 6.5 \%$, or (iii) $\mathrm{HbA} 1 \mathrm{c} 6.5 \%$ confirmed by $\mathrm{FG} \geq 126 \mathrm{mg} / \mathrm{dL}$ cohort.

Results: Overall, 390 women (285 HIV+, median age 43 years; 105 HIV-, median age 37 years) were enrolled between 2003-2006. Over half of all women were African American. Using DM_C, DM prevalence rates were 5.6\% and $2.8 \%$ among HIV+ and HIV- women, respectively. Among HIV+ women, adding DM_OGTT to DM_C increased DM prevalence from $5.6 \%$ to $7.4 \%$, a $31 \%$ increase in the number of diabetes cases diagnosed $(p=0.02)$. In HIV- women, no additional cases were diagnosed by DM-OGTT.

Conclusion: In HIV+ women, OGTT identified DM cases that were not identified by a standardized clinical definition. Further investigation is needed to determine whether OGTT should be considered as an adjunctive tool for DM diagnosis in the setting of HIV infection.
\end{abstract}

Keywords: HIV; Women; Diabetes mellitus; Oral glucose tolerance test

\section{Introduction}

Since effective antiretroviral therapy (ART) has prolonged life expectancy among HIV-infected (HIV+) persons, non-AIDS events have become increasingly important causes of morbidity and mortality [1]. HIV+ persons on ART may have up to four-fold increased risk for diabetes mellitus (DM) compared to HIV-uninfected (HIV-) persons, with $2.5-7.0 \%$ reported DM prevalence rates [2,3]. Traditional DM risk factors such as older age, obesity, family history, black, Asian or Hispanic ethnicity $[1,4]$ and HIV-related parameters (including ART exposure) have been associated with DM incidence in HIV+ persons [5,6].

Patients with undiagnosed/under-treated DM are at increasing risk for cardiovascular disease (CVD) due to the micro- and macrovascular injuries that progressively develop. HIV+ persons are also at increased CVD risk [7]. This, combined with the elevated risk of glucose disorders and rapid aging of the HIV+ patient population, amplifies the need for early and accurate DM diagnosis to minimize CVD risk in this population.

The American Diabetes Association (ADA) guidelines recommend fasting plasma glucose (FPG), glycosylated hemoglobin (HbAlc) and two-hour, 75g oral glucose tolerance testing (OGTT) for DM diagnosis [8]. In HIV+ persons, HbA1C may underestimate glycemia [9-11], possibly as a result of low-grade hemolysis $[10,11]$. OGTT has not commonly been used for DM diagnosis in large HIV+ cohorts $[2,3]$, likely due to a combination of cost, performance time, low reproducibility and high intra-individual variability [12]. Nevertheless, in the general population, OGTT has been reported to improve DM diagnosis in older individuals [13] and to be more predictive of CVD than FPG [14]. Overall, controversies regarding the optimal diagnostic algorithm for DM in HIV+ persons persist.

We examined the prevalence and incidence of DM in participants of the Women's Interagency HIV Study (WIHS) over five years of followup and aimed to determine the utility of OGTT for DM diagnosis in women with and without HIV. The WIHS has previously demonstrated that while HIV-infected women have an increased DM risk [5,6,15], a DM diagnosis made without any confirmatory criteria overestimated DM incidence. By contrast, the use of confirmatory criteria (including an elevated $\mathrm{A} 1 \mathrm{C}$ ) increased the diagnostic accuracy and slightly attenuated the magnitude of the association otherwise observed between HIV and DM [15]. We now build upon our prior work by investigating how the use of OGTT might impact on DM prevalence and incidence in HIV+ and HIV- women compared to when DM is diagnosed using confirmatory criteria including AIC.

*Corresponding author: Sophie Seang, University of California, Los Angeles 11075 Santa Monica Blvd, te 100, Los Angeles, CA 90025, USA, Tel: (310) 5571891; Fax: (310) 557-1899; E-mail: sophie.seang@yahoo.fr

Received December 17, 2015; Accepted February 15, 2016; Published February 20, 2016

Citation: Seang S, Lake JE, Tian F, Anastos K, Mardge H Cohen, et al. (2016) Oral Glucose Tolerance Testing identifies HIV+ infected women with Diabetes Mellitus (DM) not captured by standard DM definition. J AIDS Clin Res 7: 545 doi:10.4172/2155-6113.1000545

Copyright: () 2016 Seang S, et al. This is an open-access article distributed under the terms of the Creative Commons Attribution License, which permits unrestricted use, distribution, and reproduction in any medium, provided the original author and source are credited. 


\section{Patients and Methods}

\section{Study population}

The WIHS is a multicenter, prospective cohort study established in 1994 to investigate the natural history of women living with or at risk for HIV. The methods and baseline characteristics of WIHS participants have been described previously [16]. Between April 2003 and March 2006, a subset of 440 WIHS women from the San Francisco, Bronx and Chicago sites were enrolled into a prospective metabolic study (MS). Exclusion criteria included: age $>65$ years old; weight $>264$ pounds and height $>6$ feet and 1 inch (as per the dual X-ray absorptiometry manufacturer criteria); pregnancy or breastfeeding in the past six months; use of female hormones, growth hormone or steroids in the last year; and HIV seroconversion in the year prior to MS entry or during follow-up. All eligible participants underwent baseline, two-year and five-year follow-up visits for metabolic disease outcomes. For two- and five-year incidence analyses, women with prevalent DM at baseline were excluded. Informed consent was obtained from all participants in accordance with the US Department of Health and Human Services guidelines and the institutional review boards of each participating institution.

\section{Outcome definitions}

DM was defined according to ADA criteria [8] and two DM groups were created: 1) DM defined by two-hour OGTT $\geq 200 \mathrm{mg} / \mathrm{dL}$ (DM_ OGTT) or 2) DM defined using a clinical definition (DM_C). The latter included any of the following criteria: (i) anti-diabetic medication use or self-reported DM confirmed by either FPG $\geq 126 \mathrm{mg} / \mathrm{dL}$ or HbA1c $\geq 6.5 \%$, (ii) FPG $\geq 126 \mathrm{mg} / \mathrm{dL}$ confirmed by a second FPG $\geq 126 \mathrm{mg} /$ $\mathrm{dL}$ or $\mathrm{HbA} 1 \mathrm{c} \geq 6.5 \%$ or (iii) HbA1c $\geq 6.5 \%$ confirmed by FPG $\geq 126$ $\mathrm{mg} / \mathrm{dL}$. Women were considered to have prevalent DM by clinical definition if they met any of the above criteria at and prior to baseline, and incident DM_C if they met any of the above criteria at two- or five-year follow-up. The primary and secondary endpoints were the number of prevalent DM cases and incident DM cases over five years, respectively, diagnosed by each DM criteria.

\section{Statistical methods}

Differences between HIV+ and HIV- women were compared using the chi-squared test for binary or categorical variables, the t-test for continuous and normally distributed variables or the Wilcoxon rank sum test for continuous variables that were not normally distributed. DM prevalence by HIV serostatus was analyzed using the chi-squared test. McNemar's test was performed to compare DM prevalence between the two outcome definitions (DM_OGTT vs. DM_C). DM incidence by HIV serostatus over five years of follow-up was analyzed using the chi-squared test. Multivariate pooled logistic regression was performed to study associations between HIV serostatus, DM and the covariates [including age, race/ethnicity, family history of DM, body composition, hepatitis $\mathrm{C}$ virus (HCV) co-infection, current smoking and menopausal status; hematocrit and mean corpuscular volume were added in DM_C model]. Covariates included in the final multivariate model were based upon either significance in the crude model or clinical consideration and/or face validity. Estimated adjusted hazard odds ratios (OR) are reported, and a $p$ value $\leq 0.05$ defined statistical significance.

\section{Results}

\section{Population}

Among 418 women with available OGTT data enrolled in the MS, 390 women (285 HIV+, $105 \mathrm{HIV-)}$ met criteria for inclusion in the analysis of DM prevalence. Baseline demographic and clinical characteristics are described in Table 1 . Over half of women were African American. HIV+ women were older (median 43 years vs. 37 years, $\mathrm{p}<0.0001)$ and more likely to be post-menopausal $(32 \%$ vs. $7 \%$, $\mathrm{p}<0.0001)$ and have HCV co-infection ( $33 \%$ vs. $15 \%, \mathrm{p}=0.0005)$ than HIV- women. Compared to HIV- women, HIV+ women also had lower body mass index (BMI), smaller hip and waist circumferences and less trunk and leg fat, but greater triglyceride levels and lower high-density lipoprotein cholesterol levels. Among HIV+ women, median CD4+ T

\begin{tabular}{|c|c|c|c|}
\hline & HIV+ & HIV- & p-value \\
\hline & $n=285$ & $n=105$ & \\
\hline Age (years) ${ }^{+}$median (IQR) & $43(38,49)$ & $37(32,44)$ & $<.0001$ \\
\hline Race & & & 0.56 \\
\hline American African & $58 \%$ & $57 \%$ & \\
\hline Hispanic & $27 \%$ & $31 \%$ & \\
\hline Caucasian/Other & $15 \%$ & $11 \%$ & \\
\hline Cohort* & & & $<.0001$ \\
\hline $1994 / 1995$ & $65 \%$ & $41 \%$ & \\
\hline $2001 / 2002$ & $35 \%$ & $59 \%$ & \\
\hline Current Smoker & $59 \%$ & $69 \%$ & 0.10 \\
\hline Post-menopausal* $^{*}$ & $32 \%$ & $7 \%$ & $<.0001$ \\
\hline DM family history & $29 \%$ & $39 \%$ & 0.06 \\
\hline Chronic HCV infection* & $33 \%$ & $15 \%$ & 0.0005 \\
\hline \multicolumn{4}{|l|}{ Body measures, median (IQR) } \\
\hline $\mathrm{BMI}\left(\mathrm{kg} / \mathrm{m}^{2}\right)^{+}$ & $27(23,31)$ & $30(26,36)$ & $<.0001$ \\
\hline Hip circumference $(\mathrm{cm})^{+}$ & $98(91,108)$ & $\begin{array}{c}105(97 \\
116)\end{array}$ & $<.0001$ \\
\hline Waist circumference $(\mathrm{cm})^{+}$ & $88(80,98)$ & $93(83,103)$ & 0.02 \\
\hline Trunk fat $(\mathrm{kg})^{+}$ & $13(9,17)$ & $16(11,22)$ & 0.0003 \\
\hline Leg fat $(\mathrm{kg})^{+}$ & $9(6,13)$ & $12(8,16)$ & $<.0001$ \\
\hline \multicolumn{4}{|l|}{ Lipids, median (IQR) } \\
\hline $\mathrm{HDL}(\mathrm{mg} / \mathrm{dL})^{+}$ & $43(35,54)$ & $50(43,63)$ & $<.0001$ \\
\hline $\mathrm{LDL}(\mathrm{mg} / \mathrm{dL})$ & $94(74,113)$ & $\begin{array}{c}101(75 \\
124)\end{array}$ & 0.07 \\
\hline $\mathrm{TG}(\mathrm{mg} / \mathrm{dL})^{+}$ & $\begin{array}{c}109(77 \\
158)\end{array}$ & $92(63,129)$ & 0.002 \\
\hline CD4+ count (/mm3), median (IQR) & $\begin{array}{c}400(257 \\
585)\end{array}$ & & \\
\hline CD4 nadir (/mm3), median (IQR) & $\begin{array}{c}247(128 \\
350)\end{array}$ & & \\
\hline HIV-1 RNA (copies/mL), median (IQR) & $\begin{array}{c}590(80 \\
8900)\end{array}$ & & \\
\hline AIDS diagnosis & $46 \%$ & & \\
\hline On ART since last visit & $61 \%$ & & \\
\hline $\mathrm{PI}$ & $55 \%$ & & \\
\hline NRTI & $99 \%$ & & \\
\hline NNRTI & $44 \%$ & & \\
\hline Years on ART, median (IQR) & $5(1,10)$ & & \\
\hline $\mathrm{PI}$ & $2.5(0,4.5)$ & & \\
\hline NRTI & $5.5(3,8)$ & & \\
\hline NNRTI & $1.5(0,3)$ & & \\
\hline
\end{tabular}

Percentage or median with interquartile range shown

${ }^{*} P<0.05$ for $X^{2}$ test comparing HIV-versus HIV+ group

$+P<0.05$ for Wilcoxon rank sum test comparing HIV-versus HIV+ group

DM: diabetes mellitus; HCV-RNA: hepatitis C virus; BMI: body mass index; HDL: high density lipoprotein; LDL: low density lipoprotein; TG : triglyceride; HAART: highly active antiretroviral therapy; Pls: protease inhibitors; NNRTIs: Nonnucleoside reverse transcriptase inhibitors; NRTI: nucleoside reverse transcriptase inhibitors.

Table 1: Baseline clinical and demographic characteristics of 390 women by HIV status. 


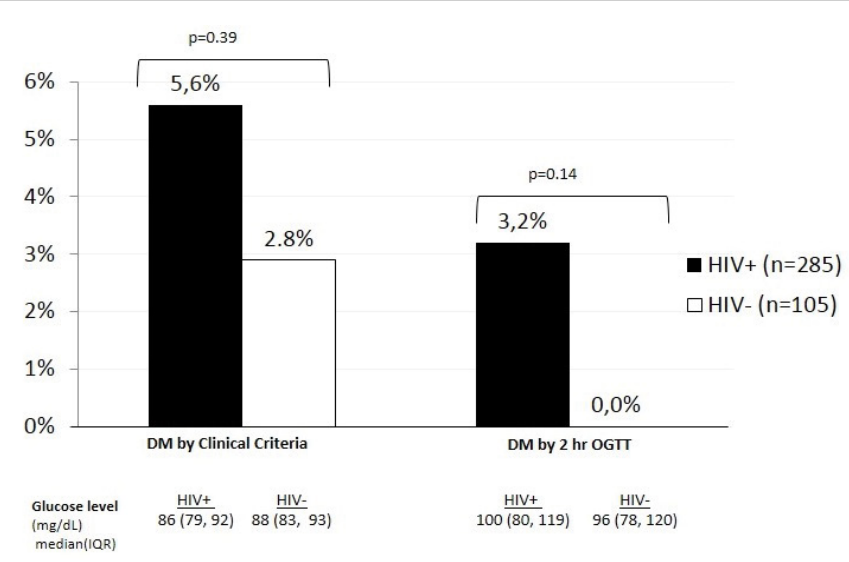

DM: diabetes mellitus; OGTT: oral glucose tolerance testing - $\mathrm{HIV}+(\mathrm{n}=285)$

$\square$ HIV- $(n=105)$

Figure 1: Prevalence of diabetes mellitus using clinical criteria and using 2-hour OGTT by HIV status.

lymphocyte and HIV-1 RNA values were 400 cells $/ \mathrm{mm}^{3}$ and 590 copies/ $\mathrm{mL}$, respectively. The cumulative years of ART exposure to the PI, NRTI and non-NRTI classes were 2.5, 5.5 and 1.5 years, respectively.

\section{Baseline DM prevalence}

Using DM_C, DM prevalences were 5.6\% and 2.8\% among HIV+ and HIV- participants, respectively. Using DM_OGTT, 3.2\% of women met DM diagnostic criteria, all of whom were HIV+ (Figure 1). HIV+ women had slightly lower median FGP levels than HIV- women (86 $\mathrm{mg} / \mathrm{dL}$ vs. $88 \mathrm{mg} / \mathrm{dL} ; \mathrm{p}=0.08$ ). After OGTT, median glucose values were slightly higher in HIV+ women but the difference was not significant (100 mg/dL vs. $96 \mathrm{mg} / \mathrm{dL} ; \mathrm{p}=0.25)$.

Among HIV+ diabetic women (diagnosed by DM_C or DM_ OGTT definition, $\mathrm{n}=21), 24 \%$ (5/21) were diagnosed only by the OGTT definition and not by the standard clinical definition. Combining OGTT and clinical criteria for DM diagnosis increased DM prevalence from $5.6 \%(16 / 285)$ to $7.4 \%(21 / 285)$ among HIV+ women, which represents a $31 \%(5 / 16)$ relative increase in the number of DM cases diagnosed vs. DM_C alone $(\mathrm{p}=0.02)$. No significant differences in clinical or demographic characteristics were reported between HIV + women diagnosed with DM by DM_C with normal OGTT $(\mathrm{n}=9)$ vs. DM_OGTT without meeting DM_C $(n=5)$. Additionally, there were no statistically significant clinical or demographic differences between non-diabetic, HIV+ women with normal OGTT $(n=230)$ and HIV+ DM_OGTT cases $(n=5)$. Among HIV- women, three DM cases were diagnosed by the DM_C definition, and all of them had normal OGTT values.

\section{DM incidence over five-year follow up and factors associated with DM incidence}

A total of 303 women (215 HIV+, 88 HIV-) were included in the five-year analysis for incident DM. The clinical characteristics were similar to the overall group described in Table 1. The five-year DM incidence rate among all women, using DM_C definition and DM_ OGTT criteria was7.0\% and $4.0 \%$, respectively ( $\mathrm{p}=0.21$ ). Among all women, greater leg fat was associated with lower odds of incident DM (DM_C: OR 0.76, 95\% CI 0.58-0.99, p=0.04; DM_OGTT: OR 0.75, 95\% CI 0.60-0.95, $\mathrm{p}=0.02$ ), and greater trunk fat with greater odds of incident DM (DM_C: OR 1.22, 95\% CI 1.04-1.43, p=0.01; DM_OGTT:
OR $1.20,95 \%$ CI 1.05-1.38, $\mathrm{p}=0.01$ ). In a subset analysis of DM cases among HIV+ women, these associations remained significant.

\section{Discussion}

In our study, nearly one quarter of $\mathrm{HIV}+$, diabetic women were diagnosed with DM by the OGTT criteria alone and not by a standardized clinical DM definition. Adding the OGTT criteria for DM diagnosis enabled a $31 \%$ relative increase in the number of prevalent DM cases diagnosed among HIV+ women. Although the overall prevalence of DM in our HIV+ population remains low (5.6\% to $7.4 \%)$, these results highlight the potential utility of OGTT among HIV+ individuals.

Previous studies have reported increased prevalence rates of DM in HIV+ individuals, but few have reported DM prevalence within the same cohort using different algorithms. Supporting our findings, Howard et al. [17] reported (in a cohort of middle age women living with or at risk for HIV infection) that $23 \%$ of women with DM defined by OGTT had FPG $<126 \mathrm{mg} / \mathrm{dL}$ and Gianoti et al. [18] reported that $4 \%$ of HIV+ men with normal FPG levels, had DM diagnosed by OGTT alone. While our conclusion was different from an earlier study in the WIHS, [19] that study did not specifically address whether the use of OGTT would detect additional cases of DM in HIV-infected adults; rather that study examined the association of HIV with prevalent DM using a DM definition that included elevated fasting plasma glucose and/or an OGTT finding consistent with DM. Because that study found little difference in DM prevalence between HIV-infected and uninfected women, they concluded that there was limited value in using OGTT in the HIV setting.

OGTT was first introduced for DM diagnosis in 1980 by the World Health Organization [20] with supporting epidemiological evidence that OGTT increased the number of DM diagnoses in the general population, particularly in patients $>50$ years old [13]. Physiologically, diabetes development involves impaired insulin sensitivity and insulin secretion that first affects post-prandial glycemia, while chronic fasting glycemia may temporarily remain normal [21]. Over time, the extent and magnitude of post-prandial hyperglycemia leads to the chronic, sustained hyperglycemia associated with DM complications [22]. This sequential progression of glucose abnormalities may explain discordant results between OGTT and FPG in clinical studies. Additionally, the absence of discordance among HIV- women may be explained by the low prevalence of DM in this group. Our findings also showed that 9 of 21 prevalent DM cases would have not been identified by OGTT alone. These results suggest that further investigation is needed to determine whether OGTT should be considered as an adjunctive tool for DM diagnosis in the setting of HIV infection including whether OGTT should be performed in HIV+ patients with specific risk factors, such as obesity, especially as OGTT is expensive and time-consuming.

To determine if the use of OGTT could be more relevant in a specific $\mathrm{HIV}+$ subgroup, we compared clinical characteristics of diabetic, HIV+ women with non-diabetic, HIV+ women. Our analysis did not show significant differences in demographic or clinical characteristics between HIV+, non-diabetic women compared to HIV+, diabetic women, a finding that may result from the low number of participants with prevalent DM. However, HIV+, non-diabetic women tended to have more leg fat than HIV+, diabetic women diagnosed either by OGTT or DM_C criteria ( $\mathrm{p}=0.10$ for both). In our multivariate model, we found that greater leg fat was significantly associated with decreased odds of DM, whereas greater trunk fat increased the odds of DM defined by OGTT definition among HIV+ women. These 
Citation: Seang S, Lake JE, Tian F, Anastos K, Mardge H Cohen, et al. (2016) Oral Glucose Tolerance Testing identifies HIV+ infected women with Diabetes Mellitus (DM) not captured by standard DM definition. J AIDS Clin Res 7: 545. doi:10.4172/2155-6113.1000545

Page 4 of 5

results are consistent with previous data in HIV+ persons in which lipodystrophy was associated with incident DM. Kosmiski et al. [23] showed that greater trunk subcutaneous adipose tissue and less leg subcutaneous adipose tissue were significantly associated with higher 2-hour glucose values in HIV+ persons. Adipose tissue is an endocrine organ with a determinant role in the secretion of the adipokines that control insulin sensitivity. In HIV+ individuals with significant lipoatrophy, perturbations of adipokine homeostasis may contribute to the development of insulin resistance [24]. Although our dual x-ray absorptiometry data cannot accurately distinguish subcutaneous from visceral adipose tissue, our results support the hypothesis that regional adiposity is related to insulin sensitivity in HIV infection. Considering the interaction between lipodystrophy and insulin sensitivity in HIV+ persons, OGTT could be a useful DM diagnostic tool in subjects with abnormal fat distribution. Additionally, in a study among HIV+ overweight women, Danoff et al. [19] reported BMI as a significant parameter for prediction of DM. Overall, DM screening among HIV+ women using OGTT could be more relevant in individuals at risk for CVD (PI exposed or with traditional CV risk factors) or with metabolic disorders.

This study has several strengths, including our ability to assess DM risk longitudinally, access to a well-characterized cohort of HIV+ and HIV- women, and the use of standardized definitions for DM. The latter is particularly important, as most previous studies in HIV have defined DM by self-report or FPG levels alone. Our study also has several limitations. First, 17\% $(n=63 / 366)$ of participants were lost-to-follow up over the five-year period. Moreover, the small number of incident DM cases in HIV+ women may have limited our ability to detect associations between risk factors and DM outcomes. Lastly, our data cannot be generalized to HIV+ men.

In conclusion, the prevalence and five-year incidence of diabetes among HIV+ and HIV- women were consistent with previously reported rates. DM diagnosis by OGTT criteria identified DM cases among HIV+ women that would not have been diagnosed by the standardized clinical definition alone. Further research is needed to identify the subgroup of HIV+ women most likely to benefit from OGTT.

\section{Acknowledgements}

Data in this manuscript were collected by the Women's Interagency HIV Study (WIHS). The contents of this publication are solely the responsibility of the authors and do not represent the official views of the National Institutes of Health (NIH).

WIHS (Principal Investigators): UAB-MS WIHS (Michael Saag, Mirjam-Colette Kempf, and Deborah Konkle-Parker), U01-Al-103401; Atlanta WIHS (Ighovwerha Ofotokun and Gina Wingood), U01-Al-103408; Bronx WIHS (Kathryn Anastos) U01-AI-035004; Brooklyn WIHS (Howard Minkoff and Deborah Gustafson), U01Al-031834; Chicago WIHS (Mardge Cohen and Audrey French), U01-Al-034993 Metropolitan Washington WIHS (Mary Young and Seble Kassaye), U01-AI-034994; Miami WIHS (Margaret Fischl and Lisa Metsch), U01-Al-103397; UNC WIHS (Adaora Adimora), U01-AI-103390; Connie Wofsy Women's HIV Study, Northern California (Ruth Greenblatt, Bradley Aouizerat, and Phyllis Tien), U01-AI-034989; WIHS Data Management and Analysis Center (Stephen Gange and Elizabeth Golub), U01-AI-042590; Southern California WIHS (Joel Milam), U01-HD-032632 (WIHS I - WIHS IV). The WIHS is funded primarily by the National Institute of Allergy and Infectious Diseases (NIAID), with additional co-funding from the Eunice Kennedy Shriver National Institute of Child Health and Human Development (NICHD), the National Cancer Institute ( $\mathrm{NCl}$ ), the National Institute on Drug Abuse (NIDA), and the National Institute on Mental Health (NIMH). Targeted supplementa funding for specific projects is also provided by the National Institute of Dental and Craniofacial Research (NIDCR), the National Institute on Alcohol Abuse and Alcoholism (NIAAA), the National Institute on Deafness and other Communication Disorders (NIDCD), and the NIH Office of Research on Women's Health. WIHS data collection is also supported by UL1-TR000004 (UCSF CTSA) and UL1TR000454 (Atlanta CTSA)

This research was also supported by National Institutes of Health grants T32 MH080634, P30 AG028748 and K23 Al110532 to JEL and K24 Al108516 to PC.

\section{References}

1. Masiá M, Padilla S, Álvarez D, López JC, Santos I, et al. (2013) Risk, predictors and mortality associated with non-AIDS events in newly diagnosed HIV-infected patients: role of antiretroviral therapy. AIDS 27: 181-189.

2. Brown TT, Cole SR, Li X, Kingsley LA, Palella FJ, et al. (2005) Antiretroviral therapy and the prevalence and incidence of diabetes mellitus in the multicenter AIDS cohort study. Arch Intern Med 165: 1179-1184.

3. De Wit S, Sabin CA, Weber R, Worm SW, Reiss P, et al. (2008) Incidence and risk factors for new-onset diabetes in HIV-infected patients: the Data Collection on Adverse Events of Anti-HIV Drugs (D:A:D) study. Diabetes Care 31: 1224 1229.

4. Butt $A A$, McGinnis $K$, Rodriguez-Barradas MC, Crystal S, Simberkoff M, et al (2009) HIV infection and the risk of diabetes mellitus. AIDS 23: 1227-1234.

5. Tien PC, Schneider MF, Cole SR, Levine AM, Cohen M, et al. (2007) Antiretroviral therapy exposure and incidence of diabetes mellitus in the Women's Interagency HIV Study. AIDS 21: 1739-1745.

6. Justman JE, Benning L, Danoff A, Minkoff H, Levine A, et al. (2003) Protease inhibitor use and the incidence of diabetes mellitus in a large cohort of HIVinfected women. J Acquir Immune Defic Syndr 32: 298-302.

7. Currier JS, Taylor A, Boyd F, Dezii CM, Kawabata H, et al. (2003) Coronary heart disease in HIV-infected individuals. J Acquir Immune Defic Syndr 33: 506 512 .

8. American Diabetes Association (2014) Standards of medical care in diabetes-2014. Diabetes Care 37: S14-80.

9. Glesby MJ, Hoover DR, Shi Q, Danoff A, Howard A, et al. (2010) Glycated haemoglobin in diabetic women with and without HIV infection: data from the Women's Interagency HIV Study. Antivir Ther 15: 571-577.

10. Diop ME, Bastard JP, Meunier N, Thévenet S, Maachi M, et al. (2006) Inappropriately low glycated hemoglobin values and hemolysis in HIV-infected patients. AIDS Res Hum Retroviruses 22: 1242-1247.

11. Polgreen PM, Putz D, Stapleton JT (2003) Inaccurate glycosylated hemoglobin A1C measurements in human immunodeficiency virus-positive patients with diabetes mellitus. Clin Infect Dis 37: e53-56.

12. Sacks DB (2011) A1C versus glucose testing: a comparison. Diabetes Care 34: 518-523.

13. Wahl PW, Savage PJ, Psaty BM, Orchard TJ, Robbins JA, et al. (1998) Diabetes in older adults: comparison of 1997 American Diabetes Association classification of diabetes mellitus with 1985 WHO classification. Lancet 352 1012-1015.

14. Qiao Q, Pyörälä K, Pyörälä M, Nissinen A, Lindström J, et al. (2002) Twohour glucose is a better risk predictor for incident coronary heart disease and cardiovascular mortality than fasting glucose. Eur Heart J 23: 1267-1275.

15. Tien PC, Schneider MF, Cox C, Karim R, Cohen M, et al. (2012) Association of HIV infection with incident diabetes mellitus: impact of using hemoglobin A1C as a criterion for diabetes. J Acquir Immune Defic Syndr 61: 334-340.

16. Barkan SE, Melnick SL, Preston-Martin S, Weber K, Kalish LA, et al. (1998) The Women's Interagency HIV Study. WIHS Collaborative Study Group. Epidemiology 9: 117-125.

17. Howard AA, Floris-Moore M, Arnsten JH, Santoro N, Fleischer N, et al. (2005) Disorders of glucose metabolism among HIV-infected women. Clin Infect Dis 40: $1492-1499$.

18. Gianotti N, Visco F, Galli L, Barda B, Piatti P, et al. (2011) Detecting impaired glucose tolerance or type 2 diabetes mellitus by means of an oral glucose tolerance test in HIV-infected patients. HIV Med 12: 109-117.

19. Danoff A, Shi Q, Justman J, Mulligan K, Hessol N, et al. (2005) Oral glucose tolerance and insulin sensitivity are unaffected by HIV infection or antiretroviral therapy in overweight women. J Acquir Immune Defic Syndr 39: 55-62.

20. Geneva WHO (1965) Diabetes Mellitus: Report of a WHO Expert Committee.

21. Golay A, Felber JP (1994) Evolution from obesity to diabetes. Diabete Metab 20: 3-14.

22. Monnier L, Colette C, Dunseath GJ, Owens DR (2007) The loss of postprandia glycemic control precedes stepwise deterioration of fasting with worsening diabetes. Diabetes Care 30: 263-269. 
Citation: Seang S, Lake JE, Tian F, Anastos K, Mardge H Cohen, et al. (2016) Oral Glucose Tolerance Testing identifies HIV+ infected women with Diabetes Mellitus (DM) not captured by standard DM definition. J AIDS Clin Res 7: 545. doi:10.4172/2155-6113.1000545

Page 5 of 5

23. Kosmiski LA, Scherzer R, Heymsfield SB, Rimland D, Simberkoff MS, et al. (2011) Association of increased upper trunk and decreased leg fat with 2-h glucose in control and HIV-infected persons. Diabetes Care 34: 2448-2453.
24. Kosmiski LA, Bacchetti P, Kotler DP, Heymsfield SB, Lewis CE, et al. (2008) Relationship of fat distribution with adipokines in human immunodeficiency virus infection. J Clin Endocrinol Metab 93: 216-224 